\title{
MATRIX BASED ON THE SECOND DERIVATIVE OF INFINITE CONVERGENT GEOMETRIC SERIES
}

\author{
Mulatu Lemma ${ }^{1 *}$ \\ *1 Department of Mathematics Savannah State University USA
}

\section{*Corresponding Author: -}

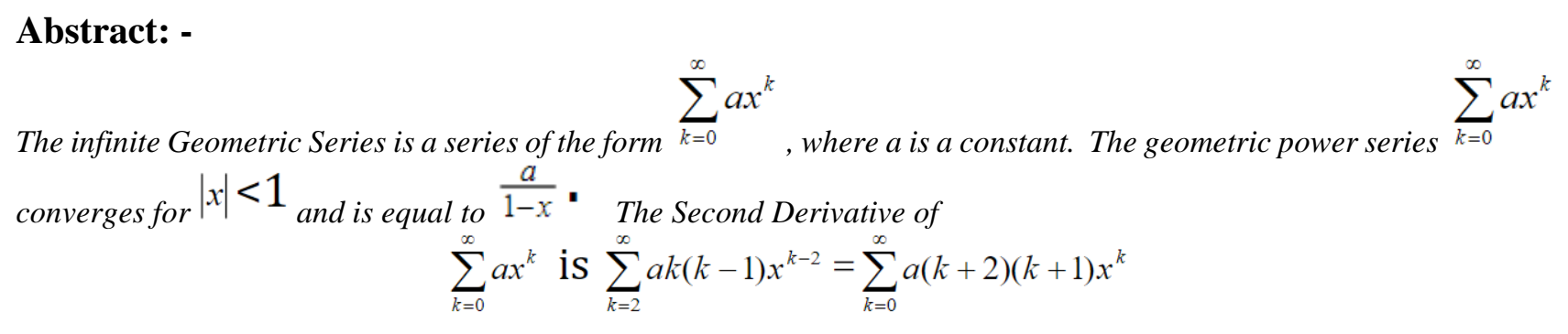

Let $t$ be sequence in $(0,1)$ that converges to 1 . The matrix based on second derivative of convergent infinite geometric series defined as

$$
\begin{aligned}
& a_{n k}=\frac{1}{2}(\mathrm{k}+2)(\mathrm{k}+1)\left(1-t_{n}\right)^{3} t_{n}{ }^{k} \text {. We denote this matrix by } \\
& \text { of geometric series. } S_{t} \text { is a sequence to sequence mapping. When } \\
& \qquad\left(S_{t} x\right)_{n}=\frac{1}{2}\left(1-t_{n}\right)^{3} \sum_{k=o}^{\infty}(k+2)(k+1) t_{n}{ }^{k} x_{k}
\end{aligned}
$$

The sequence $S_{t} x$ is called the $S_{t}$-transform of the sequence $x$.

The purpose of this research is to investigate the effect of applying $S_{t}$ to convergent sequences, bounded sequences, divergent sequences, and absolutely convergent sequences. We considering and answer the following interesting main research questions. 


\section{Research Questions.}

(1) What is the domain of $\mathrm{t}$ for which $\mathrm{S}_{t}$ maps convergent sequence into convergent sequence?

(2) What is the domain of $t$ for which the $S_{t}$ maps absolutely convergent sequence into absolutely convergent sequence?

(3) Does $S_{t}$ maps unbounded sequence to convergent sequence?

(4) Does $S_{t}$ maps divergent sequence to convergent sequence?

(5) How is the strength of the $S_{t}$ comparing to the identity matrix?

\section{Notations and Background Materials}

$\mathrm{W}=\{$ the set of all complex sequences $\}$

$\mathrm{c}=\{$ the set of all convergent complex sequences $\}$

$$
\begin{aligned}
& c(A)=\{\mathrm{y}: \mathrm{Ay} \in \mathrm{C}\} \\
& l=\left\{\mathrm{y}: \sum_{k=0}^{\infty}\left|y_{k}\right|<\infty\right\} \\
& l(A)=\{\mathrm{y}: \mathrm{Ay} \in l\}
\end{aligned}
$$

Definition 1: A matrix A is an $x-y$ matrix if the image Au of $u$ under the transformation $A$ is in $Y$ wherever $u$ is in $x$.

\section{Regular Matrix}

A matrix is regular if $\lim _{n \rightarrow \infty} Z_{n}=\mathrm{a} \Rightarrow \lim _{n \rightarrow \infty}(A X)_{n}=\mathrm{a}$. That is a sequence $\mathrm{Z}$ is convergent to $\mathrm{A} \Rightarrow$ the $\mathrm{A}$-transform of $\mathrm{Z}$ also converses to a.

\section{The Sliverman-Toeplitz Rule}

We state the following famous Sliverman-Toeplitz Rule as Proposition I without proof and apply it.

Proposition I: A matrix $\mathrm{A}=\left(a_{n}, k\right)$ is regular if and only if

$$
\begin{aligned}
& \text { (i) } \lim _{n \rightarrow \infty} a_{n, k}=0 \text { for each } \mathrm{k}=0,1, \ldots, \\
& \text { (ii) } \lim _{n \rightarrow \infty} \sum_{k=0}^{\infty} a_{n, k}=1 \text {, and } \\
& \text { (iii) } \sup _{n}\left\{\sum_{k=0}^{\infty}\left|a_{n ; k}\right|\right\} \leq M<\infty \text { for some } M<0 .
\end{aligned}
$$

\section{The Main Results}

Theorem 1: The $\mathrm{S}_{t}$ matrix is a regular matrix for all $\mathrm{t}$.

Proof: We use proposition 1, to prove the theorem. Note that

$$
\begin{aligned}
& \text { (1) } \lim _{n \rightarrow \infty} a_{n, k}=\lim _{n \rightarrow \infty} \frac{1}{2}(\mathrm{k}+2)(\mathrm{k}+1)\left(1-t_{n}\right)^{3} t_{n}{ }^{k}=0 \\
& \text { (2) } \lim _{n \rightarrow \infty} \sum_{k=0}^{\infty} a_{n k}=\lim _{n \rightarrow \infty} \frac{1}{2} \sum_{k=0}^{\infty}(k+2)(k+1) t_{n}{ }^{k}\left(1-t_{n}\right)^{3}= \\
& \lim _{n \leftarrow \infty} \frac{1}{2}\left(1-t_{n}\right)^{3} \sum_{k=0}^{\infty}(k+2)(k+1) t_{n}{ }^{k}=\frac{\left(1-t_{n}\right)^{3}}{\left(1-t_{n)}\right)^{3}}=1 \text { and } \\
& \text { (3) } \operatorname{Sup}_{n} \sum_{k=0}^{\infty} a_{n, k}=1
\end{aligned}
$$

Hence by Proposition I, the matrix $S_{t}$ is a regular matrix. Thus, the matrix $\mathrm{S}_{t}$ maps all convergent sequences into convergent sequences and we can say that the matrix $S_{t}$ a c-c matrix.

Remark 1: The $\mathrm{S}_{t}$ matrix maps a bounded sequence into a convergent sequence as shown by the following example. This shows that the $\mathrm{S}_{t}$ matrix is stronger than the identity matrix or $\mathrm{c}\left(\mathrm{S}_{t}\right)$ is larger than $\mathrm{c}$.

Example 1: Consider the bounded sequence given by $x_{k}=(-1)^{k}$

$$
\text { Then } \begin{aligned}
\left(S_{t} x\right)_{n}= & \frac{1}{2}\left(1-t_{n}\right)^{3} \sum_{k=0}^{\infty}(k+2)(k+1)\left(t_{n}\right)^{k}(-1)^{k} \\
& =\frac{1}{2}\left(1-t_{n}\right)^{3} \sum_{k=0}^{\infty}(k+2)(k+1)\left(-t^{n}\right)^{k}
\end{aligned}
$$


Remark 2: The $S_{t}$ matrix maps also a divergent sequence $\mathrm{x}$ into a convergent sequence as shown by the following example.

Example 2: Consider the unbounded sequence given by $\mathrm{x}$ defined by $x_{k}=(-1)^{k}(k+3)$. Note that

\section{Knopp-Lorentz Thorem}

$$
\begin{aligned}
& \begin{aligned}
\left(S_{t} x\right)_{n} & =\frac{1}{2} \sum_{k=0}^{\infty}\left(1-t_{n}\right)^{3} t_{n}^{k}(-1)^{k} x_{k}(k+2)(k+1) \\
& \left.=\frac{1}{2}\left(1-t_{n}\right)^{3} \sum_{k=0}^{\infty} t_{n}^{k}(-1)^{k}\right)(k+3)(k+2)(k+1) \\
& =\left(1-t_{n}\right)^{3} \sum_{k=0}^{\infty}\left(-t_{n}\right)^{k}(k+3)(k+2)(k+1) \\
& =\frac{3\left(1-t_{n}\right)^{3}}{\left(1+t_{n}\right)^{4}}
\end{aligned} \\
& \text { Now, } \lim _{n \rightarrow \infty}\left(S_{t} x\right)_{n}=\lim _{n \rightarrow \infty} \frac{\left(1-t_{n}\right)^{3}}{\left(1+t_{n}\right)^{4}}=0 \\
& \text { Hence } S_{t} x \in C .
\end{aligned}
$$

The Matrix $A$ is an $\ell-\ell_{\text {matrix if and only if there exists a number }}$ $M>0$ such that for every $k$,

$$
\sum_{n=0}^{\infty}\left|a_{n k}\right| \leq M
$$

Theorem 2: $S_{t}$ is $\ell-\ell \Leftrightarrow(1-t)^{3} \in \ell$

\section{Lemma 1:}

Proof: We use the Knopp-Lorentz Rule.

$$
S_{t \text { is } \ell-\ell_{\text {matrix }}} \Rightarrow(1-t)^{3} \in \ell .
$$

$$
\begin{aligned}
S_{t_{\text {is }}} \ell-\ell & \Rightarrow \sum_{n=0}^{\infty}\left|\left(1-t_{n}\right)^{3} t_{n}^{k}\right| \leq M \\
& \Rightarrow \sum_{n=0}^{\infty}\left|\left(1-t_{n}\right)^{3}\right| \leq M_{\text {(for k }=0)} \\
& \Rightarrow(1-t)^{3} \in \ell
\end{aligned}
$$

\section{Lemma 2:}

Proof: We use the Knopp-Lorentz Rule

$$
(1-t)^{3} \in \ell \Rightarrow S_{t_{\text {is an }}} \ell-\ell_{\text {matrix }}
$$

Now Theorem 2 follows by Lemmas $1 \& 2$.

$$
\begin{aligned}
\sum_{n=0}^{\infty}\left|a_{n k}\right| & \leq \sum_{n=0}^{\infty}\left|\left(1-t_{n}\right)^{3} t_{n}^{k}\right| \\
& \leq \sum_{n=0}^{\infty}\left(1-t_{n}\right)^{3} \leq M_{\text {for some } \mathrm{M}>0 \text { as }}(1-t)^{3} \in \ell .
\end{aligned}
$$

Corollary 1. $\arcsin (1-t)^{2} \in 1 \Leftrightarrow S_{t}$ is an 1-1 matrix.

Proof: The corollary easily follows using Theorem 2 and the following basic inequality.

$$
(1-t)^{3} \leq \arcsin (1-t)^{3} \leq \frac{(1-t)^{3}}{\sqrt{1-(1-t)^{3}}} .
$$

Theorem $3 \frac{-1}{\ln \left(1-t_{n}\right)} \in \mathrm{I} \Rightarrow \mathbf{S}_{t}$ is an 1-1 matrix.

Proof. Note that: 


$$
\begin{aligned}
\left(1-t_{n}\right)^{3} & \leq\left(1-\mathrm{t}_{n}\right):=\left(\sum_{k=0}^{\infty} t_{n}{ }^{k}\right)^{-1} \\
& \leq\left(\sum_{k=0}^{\infty} \frac{1}{k+1} t_{n}{ }^{k}\right)^{-1} \\
& =\left(\sum_{k=0}^{\infty} t_{n}{ }^{k}\left(\int_{0}^{1} V^{k} d V\right)\right)^{-1} \\
& =\left(\sum_{k=0}^{\infty}\left(\int_{0}^{1} t_{n}{ }^{k} V^{k} d V\right)\right)^{-1} \\
& =\left(\int_{0}^{1} d V\left(\sum_{k=0}^{\infty}\left(t_{n} V\right)^{k}\right)\right)^{-1}
\end{aligned}
$$

The Interchanging of the Integral and summation is legitimate as the power series

$$
\sum_{k=0}^{\infty}\left(V t_{n}\right)^{k} \text { converges absolutely and uniformly for } 0 \leq V t_{n} \leq 1 \text {. Hence we have, }
$$

$$
\begin{aligned}
\left(1-t_{n}\right)^{3} & \leq 1-\mathrm{t}_{n} \leq\left(\int_{0}^{1} \frac{d V}{1-V t_{n}}\right)^{-1} \\
& =\left(\frac{-1}{t_{n}}\left(\ln \left(1-t_{n}\right)\right)^{-1}\right. \\
& \leq \frac{-1}{\ln \left(1-t_{n}\right)}
\end{aligned}
$$

The hypothesis that $\frac{-1}{\ln (1-t)} \in \mathrm{I} \Rightarrow(1-\mathrm{t})^{3} \in \mathrm{I}$ and hence by Theorem 2, $\mathrm{S}_{t}$ is 1-1.

Remark 3. An 1-1 $S_{t}$ matrix maps a bounded sequence into $l$ as shown by the following example. This shows that the $S_{t}$ matrix is stronger than the identity matrix in the $l$ - $l$ setting or $1\left(S_{t}\right)$ is larger than $l$.

\section{Example 3.}

Assume the $S_{t}$ matrix is $l-l$ and consider the bounded sequence given by $x_{k}=(-1)^{k}$

$$
\text { Then } \begin{aligned}
\left(S_{t} x\right)_{n}= & \frac{1}{2}\left(1-t_{n}\right)^{3} \sum_{k=0}^{\infty}(k+1)(k+2)\left(t_{n}\right)^{k}(-1)^{k} \\
& =\frac{1}{2}\left(1-t_{n}\right)^{3} \sum_{k=0}^{\infty}(k+2)(k+1)\left(-t^{n}\right)^{k} \\
& =\left(1-t_{n}\right)^{3} \frac{1}{\left(1+t_{n}\right)^{3}} \\
& \leq\left(1-t_{n}\right)^{3}
\end{aligned}
$$

Now the $S_{t}$ matrix is $\mid-l \Rightarrow(1-t)^{3} \in \mathrm{I}$, by Theorem 2, and hence $S_{t} x \in l$.

Remark 4: An 1-1 $S_{t}$ matrix maps unbounded sequence into $l$ as shown by the following example.

Example 4: Assume $S_{t}$ is an $l-l$ matrix and consider the unbounded sequence given by $x_{k}=(-1)^{k}(k+3)$. Note that 


$$
\begin{aligned}
\left(S_{t} x\right)_{n} & ==\frac{1}{2}\left(1-t_{n}\right)^{3} \sum_{k=0}^{\infty} t_{n}^{k}(-1)^{k}(k+3)(k+2)(k+1) \\
& =\frac{1}{2}\left(1-t_{n}\right)^{3} \sum_{k=0}^{\infty}\left(-t_{n}\right)^{k}(k+3)(k+2)(k+1) \\
& =\frac{\left(1-t_{n}\right)^{3}}{\left(1+t_{n}\right)^{4}} \\
& \leq\left(1-t_{n}\right)^{3}
\end{aligned}
$$

Important Dedication: This paper is dedicated to my Great wife Mrs. Aster Debebe for her extraordinary support on my research activities. I am able to be a good mathematician because of her. Thank you Aster.

\section{Acknowledgements:}

Special thanks to Dawit Getchaw, Eneye Negassa, Samea Mulatu, Abyssinia Mulatu, Sara Worku, Lela Mikre, Benni Fekadu and Akalewolde Wegayhu.

\section{Reference:}

[1].M. Lemma, Logarithmic transformations into l1, Rocky Mountain J. Math. 28 (1998), no. 1, 253-266. MR 99k:40004. Zbl 922.40007.. 